Supporting Information to:

\title{
Differential Isotope Labeling of Glycopeptides for Accurate Determination of Differences in Site-Specific Glycosylation
}

\author{
M. Pabst ${ }^{\# 1}$, I. Benešová ${ }^{\# 1,2}$, S. R. Fagerer ${ }^{1}$, M. Jacobsen ${ }^{1}$, K. Eyer $^{1}$, G. Schmidt ${ }^{3}$ R. Steinhoff ${ }^{1}$,
} J. Krismer ${ }^{1}$, F. Wahl ${ }^{4}$, J. Preisler ${ }^{2,5}$ and R. Zenobi ${ }^{1}$

${ }^{1}$ Department of Chemistry and Applied Biosciences, ETH Zurich, 8093 Zurich, Switzerland

${ }^{2}$ Department of Chemistry, Masaryk University, 62500 Brno, Czech Republic

${ }^{3}$ Department of Biosystems Science and Engineering (D-BSSE), ETH Zurich, 4058 Basel, Switzerland

${ }^{4}$ Sigma-Aldrich Chemie GmbH, 9470 Buchs, Switzerland

${ }^{5}$ Central European Institute of Technology (CEITEC), Masaryk University, 62500 Brno, Czech Republic

*Correspondence to: zenobi@org.chem.ethz.ch

${ }^{\#}$ These authors contributed equally

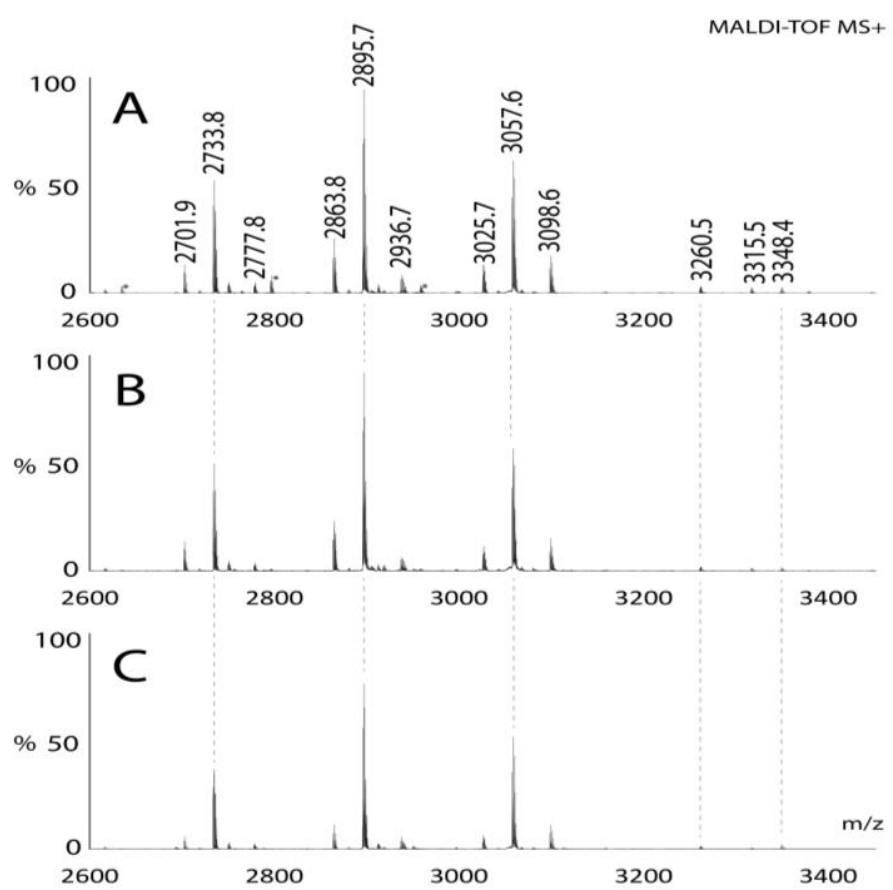

Figure S-1: Direct MALDI-MS analysis of succinic anhydride labeled glycopeptides from the labelling reaction mixture (SA0, with mass shift of +100.01 Da over the unlabeled glycopeptides). ( $\mathrm{A}=100 \mu \mathrm{g}, \mathrm{B}=25 \mu \mathrm{g}$ and $\mathrm{C}=5 \mu \mathrm{g}$ ). Labelling could be directly followed by MALDI-MS and for all samples tested, the labeling efficiency was above $90 \%$ after 1 hour of reaction (based on MALDI-MS signal). The $[\mathrm{M}+\mathrm{H}]^{+}$peaks with the $\mathrm{m} / \mathrm{z}$ of 2733.8 , 2895.7 and 3057.6 represent the major IgG glycopeptides with the glycofroms G0F (GnGnF), G1F (AgnF/GnAF) and G2F (AAF) (proglycan nomenclature: http://www.proglycan.com/index.php?page=pga_nomenclature). The IgG2 glycopeptides are visible as a separate series with -32 Da compared to the IgG1 glcyopeptides. 
*(Peaks originating from unlabelled glycopeptide portions are indicated by an asterisk)

Effect of the SA-label on ionization efficiency in MALDI. The labeling process introduces an additional negative charge on the peptide backbone. Because positive reflectron mode proved to give lower detection limits for the glycopeptides analysed compared to negative reflectron mode, this could potentially lead to loss of sensitivity. Therefore, a simple experiment to exclude a significant lack of ionization potential for labeled glycopeptides was performed. A tryptic digest of a single sample was split into two aliquots. The first was labeled with SA0 while the second was incubated only in the labeling reaction mixture without addition of the labeling agent. Both samples were then pooled and subjected to ZICHILIC SPE. The drop in signal intensity for the labeled glycopeptides was rather small, although a few percent in the labeling fractions remained unlabeled and contributed to the signal of the unlabeled fraction. Despite the presence of an additional negatively charged group, the positive ion mode was still superior over the negative one (data not shown).

\section{Precision}

\section{IgG 1}

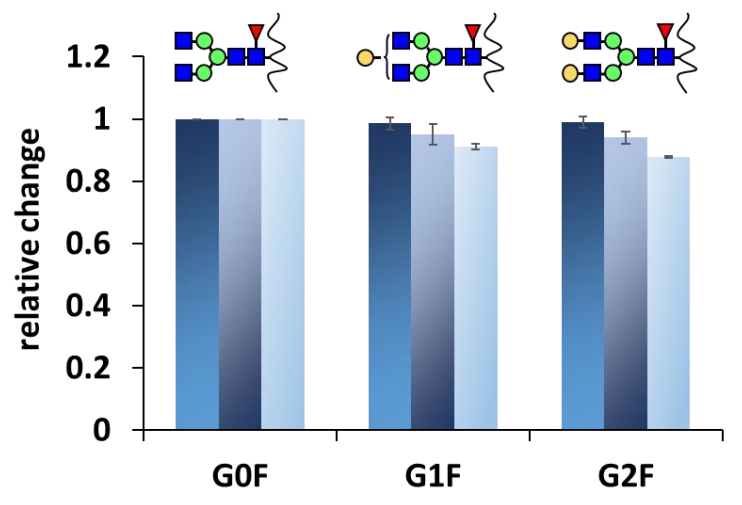

IgG 2

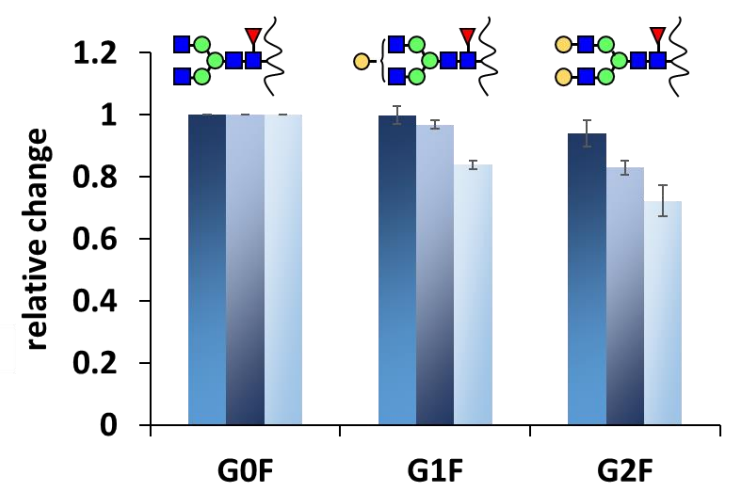

Figure S-2: The graph shows the comparison between different polyclonal $\operatorname{IgG}$ samples analysed by MALDI-MS in positive ionisation mode and DHB as matrix (IgG1 left graph, IgG2 right graph) obtained from three separate MALDI-MS analysis, including error bars which representing standard deviations for experiments. The first pair (left column) shows the control batch (same batch labelled once with D0 and once with D8), the second pair (middle column) compares two different Privigen batches and the third pair (right column) compares a Privigen batch with polyclonal IgG from a chemical supplier. 


\section{Reversed phase analysis and deuterium retention effect}

By analyzing the above mentioned validation mixture by nano reversed phase liquid chromatography, separate profiles were obtained for the individual IgG subclasses. The employed reversed phase and buffer system resulted in a slight separation of individual glycoforms, where mainly the sialylated part eluted a few seconds after the neutral portions. Furthermore, the N-terminal succinic acid slightly increased the retention on the reversed phase (C18) column. The label provides therefore additional retention for small or hydrophilic glycopeptides, which can suffer from a weak binding otherwise.(1)

Noteworthy, deuterium containing stable isotope labels show a secondary isotope effect when analysed by reversed phase liquid chromatography.(2) This becomes apparent in a slightly higher retention of the deuterium isotope labeled peptide, and has to be considered when comparing the intensities or areas of the analyte peaks. As shown in the figure below this was also found for the SA8 label used in our experiments and was considered when evaluating the summarized ion intensities.

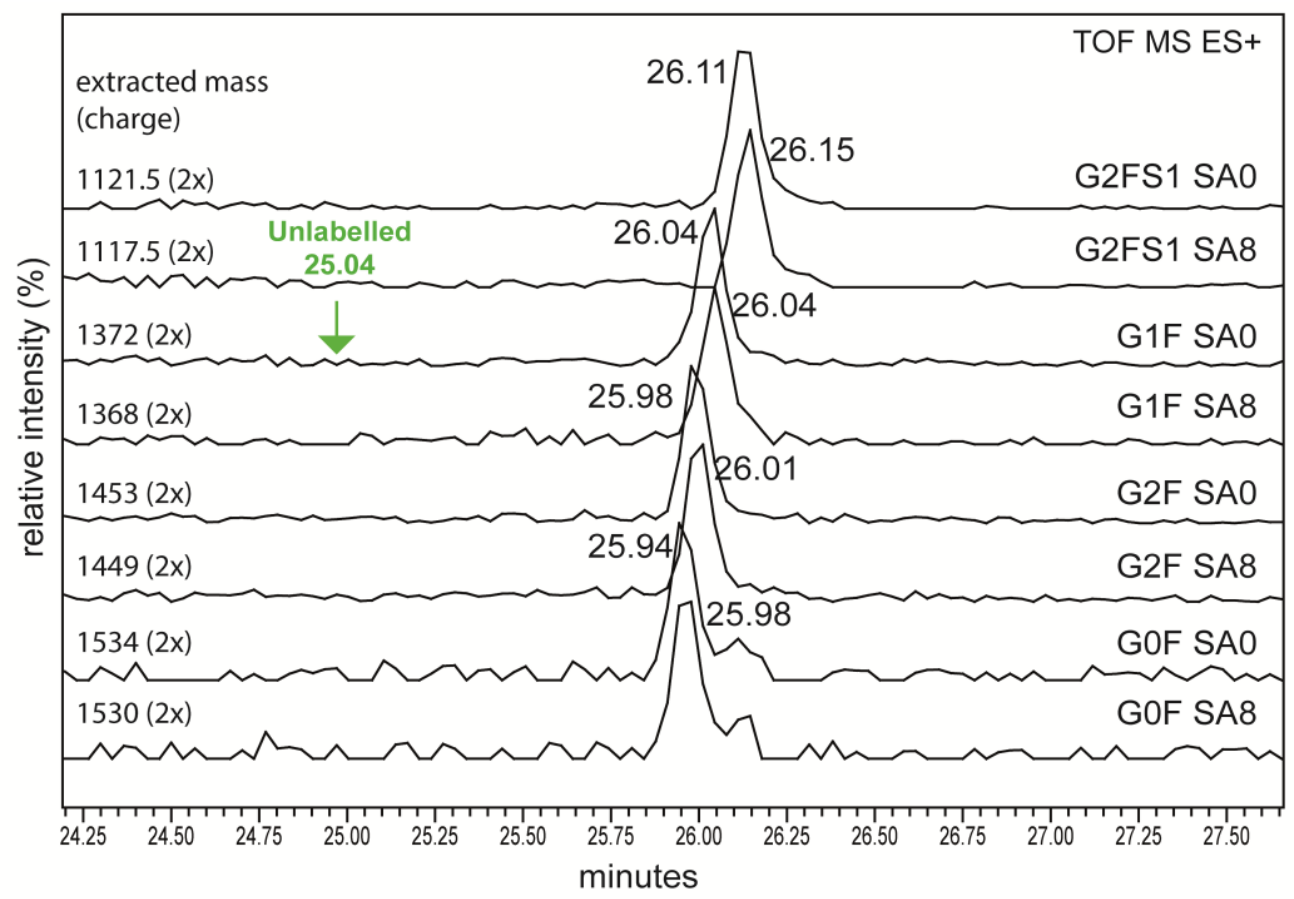

Figure S-3: Stable isotope labeled glycopeptides show a deuterium retention effect (retention difference between the SA0 and SA8 species, separation was performed on a reversed phase C18 column) which became apparent in the slightly higher retention of the deuterium isotope labeled peptide (SA8). The overall increase in retention was found to be approximately 1-2 seconds for the employed gradient. The green arrow indicates the elution position of the unlabeled counterpart of the G1F glycopeptide. 


\section{MALDI-MS analysis for comparing different polyclonal IgG samples}

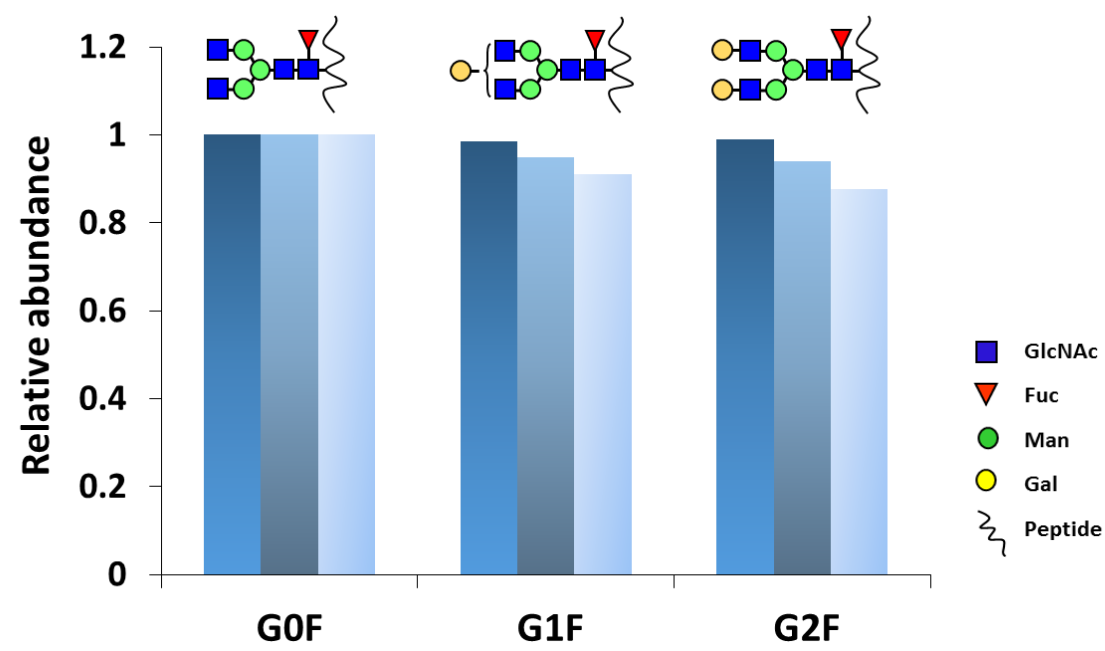

Figure S-4: The graph shows small differences in the degrees of galactosylation present on the Fc regions of IgG1 from different human polyclonal IgG sources analysed per MALDIMS. The left bar represents Privigen (used also in its SA8 form as internal standard for the other two samples), the middle bar shows the levels of an different Privigen batch, and the right bar shows a human polyclonal IgG sample obtained from a chemical supplier. For each glycoform the signal intensity ratio with its internal SA8 standard was calculated and then normalised to the intensity of the $\mathrm{G} 0 \mathrm{~F}(\mathrm{GnGnF})$ intensity ratio. 


\section{MALDI-MS analysis of samples with a modified degree of sialylation}
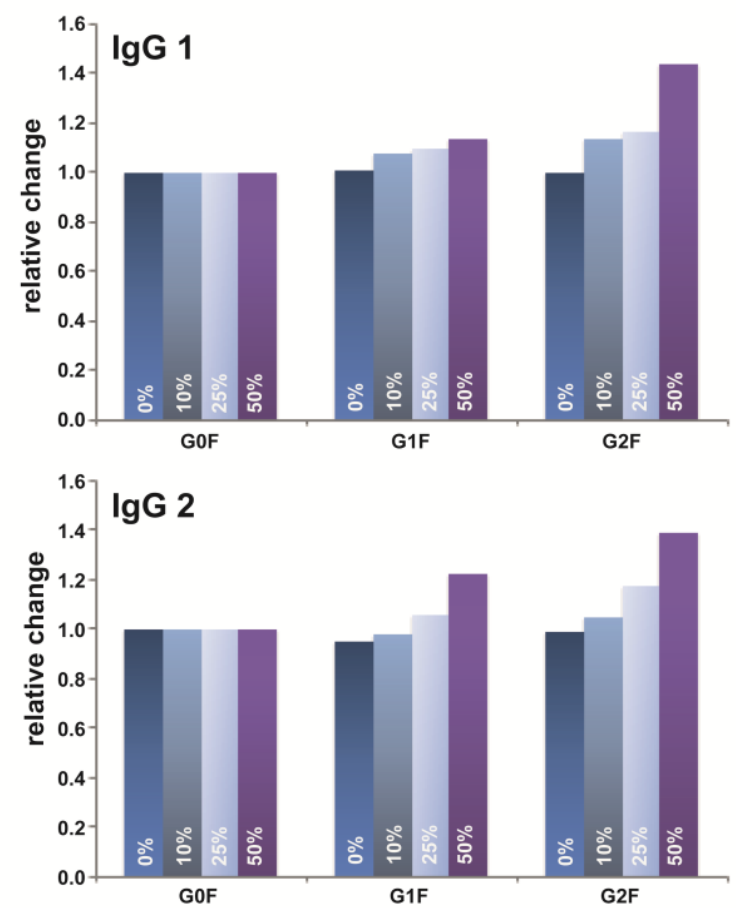

Figure S-5: MALDI-MS analysis of the test series differentiating in the degree of sialylation. For each glycoform the signal intensity ratio with its internal SA8 standard was calculated and then normalised to the intensity of the G0F glycoform.

The sialylated glycans were barely detected by the direct MALDI-MS approach. Nevertheless the reduced sialylation resulted in an increase in the G1F and G2F abundance. The left sample is the control sample (no change in sialylation), the middle left sample has a $10 \%$ change in sialylation, the middle right sample has a $25 \%$ change in sialylation and the right sample corresponds to a $50 \%$ change in sialylation. 


\section{Nano-LC-ESI-MS analysis of samples with modified degrees of sialylation}

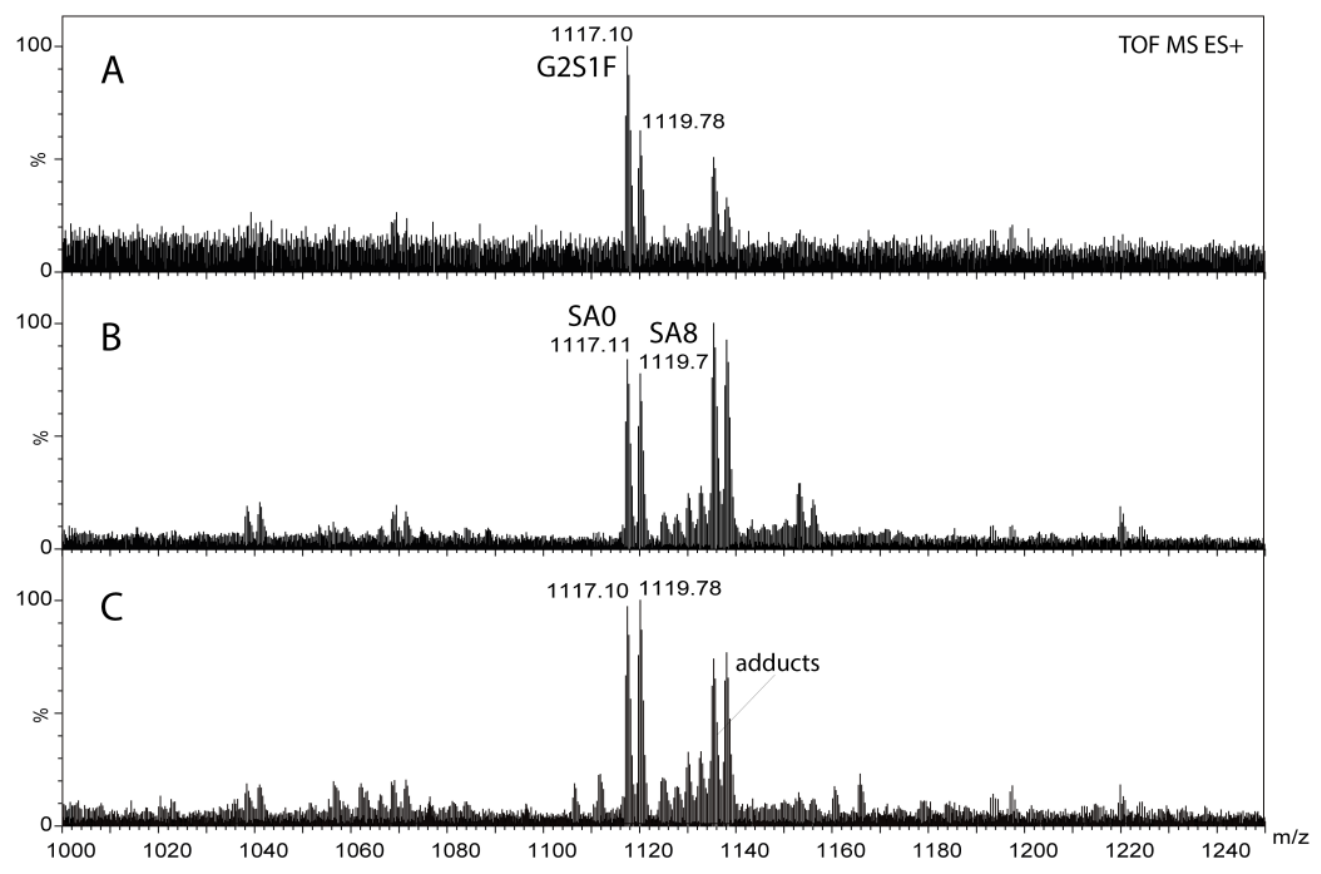

Figure S-6: The spectra A-C show a series of different degrees of sialylation, where spectrum A shows $50 \%$ difference in sialylation, spectrum B shows $10 \%$ difference in sialylation and spectrum $\mathrm{C}$ a mixture of both untreated samples. In each case, the untreated control sample was labelled with SA0 and the neuraminidase treated sample was labelled with SA8.

All three graphs show a spectrum summarized over the elution range of the glycopeptide G2F1S. The corresponding $(\mathrm{M}+3 \mathrm{H})^{3+}$ ion corresponding to the single sialylated Fc IgG1 glycopeptide is annotated in each spectrum. 


\section{Nano-LC-ESI-MS analysis of desialylated sample}

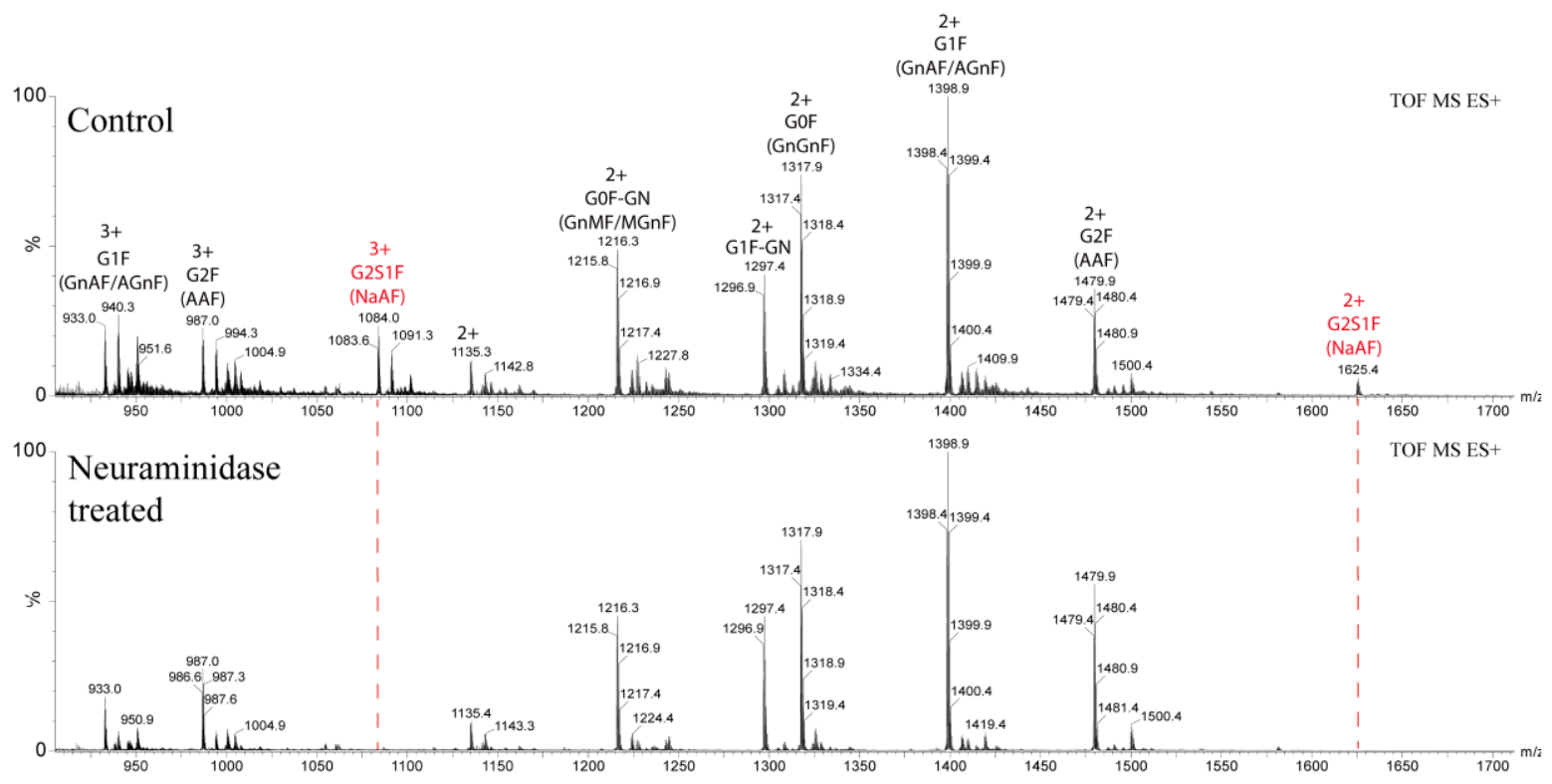

Figure S-7: The upper summarized spectrum shows the glycoprofile from the control sample where the lower one represents the glycoprofile spectrum from the neuraminidase treated aliquot, as obtained after LC-ESI-MS analysis. The neuraminidase treated samples showed no indication of sialylated species, as seen by the absence of the peaks for G2S1F (doubly and triply positively charged) in the lower spectrum (highlighted in red).

As observed in this particular run, glycopeptides tend to undergo in source fragmentation, to variable extend depending on the charge state of the glycopeptide, on the peptide itself and on the glycoforms. Stable isotope labelled internal standards can correct this bias since both standard and analyte would undergo the same in/post source decay. A prior orthogonal characterized internal standard, e.g. by glycan release and fluorescence labelling, would then allow to determine the absolute stoichiometry of investigated glycoforms. 


\section{References for Supporting information:}

1. Pabst, M.; Chang, M.; Stadlmann, J.; Altmann, F., Glycan profiles of the 27 N-glycosylation sites of the HIV envelope protein CN54gp140. Biol Chem 2012, 393, (8), 719-30.

2. Valleix, A.; Carrat, S.; Caussignac, C.; Leonce, E.; Tchapla, A., Secondary isotope effects in liquid chromatography behaviour of $2 \mathrm{H}$ and $3 \mathrm{H}$ labelled solutes and solvents. J Chromatogr A 2006, 1116, (1-2), 10926. 